\section{Self-maintenance of weathering test systems}

The calibrating sensors developed by Atlas Material Testing Techtain their systems themselves, which guarantees reliable test results at all times. The new combination sensors calibrate both the light (radiation) and the temperature at the same time. Recording both measurements simultaneously under identical conditions results in higher levels of precision and a significant time saving. Easyto-use sensor adapters guarantee that the correct measuring position is used and ensure that the results of the calibration process are reliable.

For more information: www.atlas-mts. de, atlas.info@ametek.de

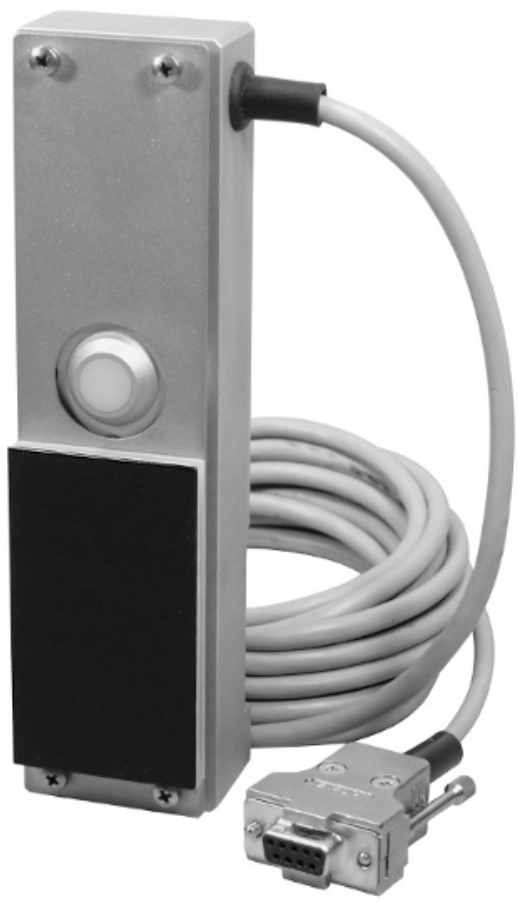

\section{Compostable adhesive system receives certification}

B ASF gained a further TÜV certificate (from DIN Cert$Z_{\mathrm{co}}$ ) for its Epotal ECO adhesive, which has already been certified as being compostable. This new certification confirms that the entire adhesive system, consisting of Epotal ECO and its accompanying curing agent, fulfils the requirements of the European standard for compostable plastics (EN 13432) and, therefore, does not present any risks to the composting process.

This biodegradable, water-based adhesive was the first to be awarded the coveted certificate for compostable additives in 2010. It is now even more effective. Higher adhesion levels can be achieved by combining the adhesive dispersion with a special curing agent. Test results show that within 70 days 90 percent of the adhesive has broken down. After decomposition it leaves no toxic residues and has no negative effects on the environment. It can be used with all common biodegradable materials and is ideal for bonding paper and manufacturing completely compostable packaging. Initial prototypes, such as coffee packaging and bags for sweets or instant soups, demonstrate its practical applications. Interest in compostable materials is growing in other areas such as the shoe, carpet, clothing and sports goods industries.

\title{
Adhesives for reliable bonding
}

ernon has a series of adhesives in its product portfolio that
can be used for thread-locking bolted joints and bonding cy-

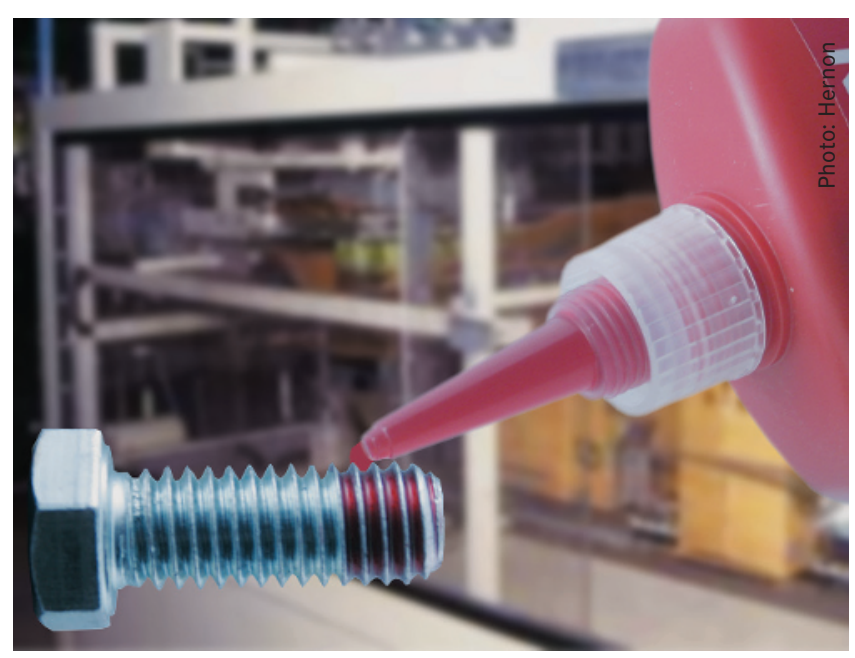

lindrical components. In Germany these products are marketed by the company 4 Advanced Technologies.

They include the anaerobic single-component adhesives from the Nuts N'Bolts product family which have different viscosities and strengths for different temperature ranges and are designed for thread-locking in a variety of applications

Another anaerobic single-component adhesive, sold under the name Cylinlock, can be used to assemble cylindrical components, such as rotors, gears and bearings. The good flow properties of these adhesives allow them to fill voids completely and prevent bolted joints or cylinders from becoming loose or detached as a result of external influences such as vibration or impacts. The adhesives are also certified in accordance with MIL-S-22473E, MILS-46163A and MIL-S-46082B.

For more information: twitt@4advancedtechnologies.de 